\begin{tabular}{|c|l|}
\hline Title & Local Spin Moment Distribution in A ntiferromagnetic Molecular Rings Probed by NMR \\
\hline Author(s) & Micotti, E.; Furukawa, Y.; Kumagai, K.; Carretta, S.; Lascialfari, A.; Borsa, F.; Timco, G. A.; Winpenny, R. E. P. \\
\hline Citation & $\begin{array}{l}\text { Physical Review Letters, 97(26), 267204 } \\
\text { https://doi.org/10.1103/PhysRevLett.97.267204 }\end{array}$ \\
\hline Issue Date & 2006-12-31 \\
\hline Doc URL & http://hdl.handle.net/2115/17195 \\
\hline Rights & Copyright $\odot$ 2006 A merican Physical Society \\
\hline Type & article \\
\hline File Information & PRL97-26.pdf \\
\hline
\end{tabular}

Instructions for use 


\title{
Local Spin Moment Distribution in Antiferromagnetic Molecular Rings Probed by NMR
}

\author{
E. Micotti, ${ }^{1,2, *}$ Y. Furukawa, ${ }^{2}$ K. Kumagai, ${ }^{2}$ S. Carretta, ${ }^{3}$ A. Lascialfari, ${ }^{1,4}$ F. Borsa, ${ }^{1}$ G. A. Timco, ${ }^{5}$ and R. E. P. Winpenny ${ }^{5}$ \\ ${ }^{1}$ Department of Physics “A. Volta” and CNR-INFM, Via Bassi 6, I-27100 Pavia, Italy \\ ${ }^{2}$ Division of Physics, Graduate School of Science, Hokkaido University, Sapporo 060-0810, Japan \\ ${ }^{3}$ Department of Physics and CNR-INFM, Universitá di Parma, I-43100 Parma, Italy \\ ${ }^{4}$ Istituto di Fisiologia Generale e Chimica Biologica, Universitá di Milano, I-20134 Milano, CNR-INFM-S3 Modena, Italy \\ ${ }^{5}$ Department of Chemistry, University of Manchester, Oxford Road, Manchester M13 9PL, United Kingdom
}

(Received 13 September 2006; published 29 December 2006)

\begin{abstract}
The NMR spectra of ${ }^{19} \mathrm{~F}$ and ${ }^{53} \mathrm{Cr}$ have been obtained at low temperatures in a heterometallic substituted antiferromagnetic (AF) ring $\mathrm{Cr} 7 \mathrm{Cd}$ with an $S=3 / 2$ ground state and compared with the spectra in a homometallic Cr8 AF ring with an $S=0$ ground state. From the analysis of the spectra one can derive directly model independent values of the staggered nonuniform distribution of the local moment in the heterometallic ring Cr7Cd. The experimental values are found to be in excellent agreement with the theoretical values calculated on the basis of an effective spin Hamiltonian which includes crystal field effects.
\end{abstract}

DOI: 10.1103/PhysRevLett.97.267204

Finite size nanoscopic systems have attracted attention for being halfway between atoms and bulk systems and thus important for their implications in fundamental physics. In this category molecular nanomagnets [1] have revealed very important features like the quantum tunneling of the magnetization (QTM) [2-4] and have been indicated to be suitable for applications like magnetic storage, magnetoelectronics, and quantum computing [5,6]. They are characterized by a strongly interacting magnetic core made of transition metal ions surrounded by an organic shell which prevents the magnetic interaction among different molecules. A particular subgroup of molecular nanomagnets, the so-called antiferromagnetic $(\mathrm{AF})$ rings $[7,8]$, has an almost coplanar ring shape with a number $N$ of transition metal ions connected by bridging ligands, which mediate nearest-neighbor superexchange interaction $J$. Because of their finite size, AF rings have a discrete energy spectrum and every state can be labeled by the quantum numbers $S$ (total spin value) and $M_{S}$ (projection along the quantization $z$ axis). The energies of the lowest lying multiplet states for a given total spin $S$ can be approximated in terms of the Lande's rule: $E(S)=$ $(P / 2) S(S+1)$, where $P=4 J / N$. An external magnetic field lifts the degeneracy of the $M_{S}$ levels thus inducing successive ground-state crossovers. Furthermore, intramolecular anisotropic interactions lead to uniaxial anisotropy and zero field splitting of the total spin multiplets.

Homonuclear AF rings like, e.g., $\mathrm{Cr} 8$ are characterized by a singlet zero field ground state with total spin $S=0$. On the other hand, in a recently synthesized subgroup of AF rings called heterometallic AF rings [9] the substitution of one of the magnetic ions with a different ion leads to a change of the ground state from $S=0$ to $S \neq 0$. As a consequence, the local spin moment in the ground state becomes redistributed in a staggered and nonuniform way. Heterometallic AF rings have been proposed as possible candidates for implementation of qubits for quantum com-
PACS numbers: 75.50.Xx, 03.67.Lx, 75.50.Ee, 76.60.-k

putation [6]. In particular, the site dependence of local moments plays a key role in the scheme proposed for obtaining time dependent qubit-qubit interactions in the presence of permanent exchange couplings [6].

With this motivation in mind we set up to derive directly the local spin moment distribution from NMR spectra. For this purpose we chose to establish the technique in one of the simplest heterometallic rings, i.e., $\mathrm{Cr} 7 \mathrm{Cd}$ where a diamagnetic $\mathrm{Cd}^{2+}$ ion with $s=0$ is replaced for a paramagnetic $\mathrm{Cr}^{3+}$ ion with $s=3 / 2$. As will be shown it is possible to map the local spin quantum reduction and its distribution caused by the spin defect in the ring and give an account of the hyperfine interactions. The model independent experimental results obtained for the local spin moment are then compared with the prediction from theoretical calculations, based on a model Hamiltonian. The excellent agreement obtained is of paramount importance to give confidence in theoretical calculations in AF heterometallic rings based on model Hamiltonians.

$\left[\mathrm{Cr}_{8} \mathrm{~F}_{8} \mathrm{Piv}_{16}\right] \cdot 0.25 \mathrm{C}_{6} \mathrm{H}_{14} \quad$ [HPiv $=$ pivalic acid, trimethyl acetic acid $\left(\mathrm{CH}_{3}\right)_{3} \mathrm{CCOOH}$ ] (in short $\mathrm{Cr} 8$ ) single crystals were prepared as described in Ref. [10]. The sample has a tetragonal structure.

$\mathrm{Me}_{2} \mathrm{NH}_{2}\left[\mathrm{Cr}_{7} \mathrm{CdF}_{8} \mathrm{Piv}_{16}\right]$ (in short $\mathrm{Cr} 7 \mathrm{Cd}$ ) single crystal $\left(1.5 \times 1.5 \times 0.3 \mathrm{~mm}^{3}\right)$ was prepared as described in Ref. [9]. The sample has a tetragonal structure with two molecules for each primitive cell.

${ }^{19} \mathrm{~F}$ and ${ }^{53} \mathrm{Cr}$ NMR spectra in $\mathrm{Cr} 7 \mathrm{Cd}$ and ${ }^{19} \mathrm{~F}$ spectra in Cr8 were performed using a pulsed NMR spectrometer at $T=1.5 \mathrm{~K}$. The spectra have been collected point by point by integrating the whole Hahn $\left(90^{\circ}-180^{\circ}\right.$ sequence) echo while sweeping the external field at the fixed Larmor frequency $\omega_{L}$.

The ${ }^{19} \mathrm{~F}$ NMR spectra at low temperature are shown in Fig. 1. In the homonuclear $\mathrm{Cr} 8$ case a single narrow and symmetric line is observed both for ${ }^{19} \mathrm{~F}$ NMR (shown in the inset of Fig. 1) and for ${ }^{1} \mathrm{H}$ NMR (not shown here) proving 


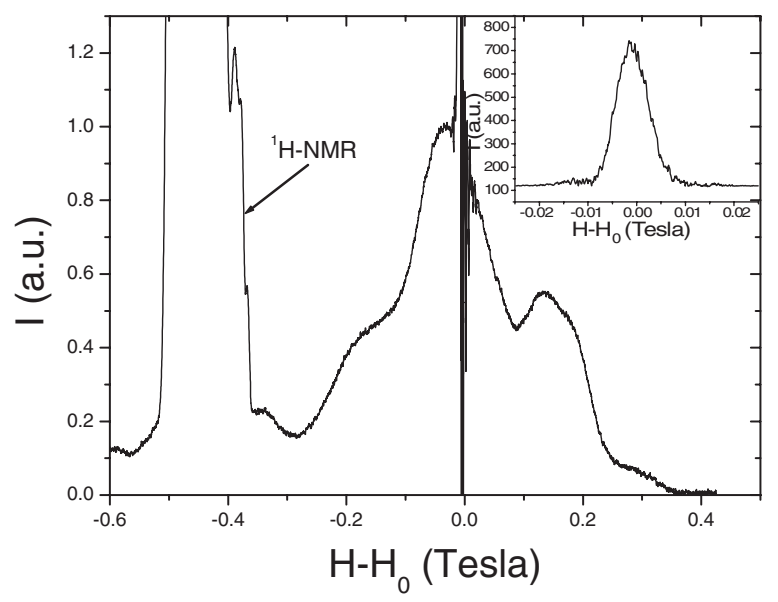

FIG. 1. ${ }^{19} \mathrm{~F}$ NMR spectrum in $\mathrm{Cr} 7 \mathrm{Cd}$ at $T=1.5 \mathrm{~K}$ and $\nu=$ $300.12 \mathrm{MHz}\left(H_{0}=7.5 \mathrm{~T}\right)$ under the external magnetic field $H$ perpendicular to the plane of the ring. The inset shows the equivalent spectrum in $\mathrm{Cr} 8$.

that the local spin moment at the $\mathrm{Cr}^{3+}$ site is $\langle s\rangle=0$ in the total spin $S=0$ ground state. On the other hand, the pronounced broadening and structure of the ${ }^{19} \mathrm{~F}$ NMR spectrum in heterometallic Cr7Cd (see Fig. 1) is the result of the hyperfine interactions with the $\langle s\rangle \neq 0$ local spin moments at the $\mathrm{Cr}^{3+}$ sites. An attempt to simulate the spectrum with the known dipolar interaction of ${ }^{19} \mathrm{~F}$ with the $\mathrm{Cr}^{3+}$ magnetic ions indicates the presence of a sizeable contribution from transferred hyperfine contact terms [11]. Thus no model independent determination of the local spin moment distribution can be obtained from ${ }^{19} \mathrm{~F}$ NMR. The ${ }^{53} \mathrm{Cr}$ NMR instead yields a direct information on the local spin moment of the magnetic ion. ${ }^{53} \mathrm{Cr}$ nucleus $(I=3 / 2)$ has low natural abundance $(9.54 \%)$ and very low sensitivity. In spite of this drawback, a weak signal was observed at high applied magnetic fields and low temperature.

Quadrupole effects of the ${ }^{53} \mathrm{Cr}(I=3 / 2)$ NMR can be disregarded since we measure the central line transition $(+1 / 2 \longleftrightarrow-1 / 2)$ which is shifted only in second order and by a negligible amount at the fields at which measurements were made. In the field range 6-8 $\mathrm{T}$ two weak signals were detected at high frequency $(60-63 \mathrm{MHz})$ which increase in frequency with increasing field as shown in Fig. 2. Another broad line was detected at a much lower frequency which shifts at lower frequency by increasing the magnetic field (see Fig. 2). The NMR signal can be observed only at high fields at $1.5 \mathrm{~K}$. In fact, the anisotropy barrier for reorientation of the spins along the uniaxial direction $z$ is only of the order of $1 \mathrm{~K}$ and a high external field directed along the $z$ axis is necessary to stabilize the local spin configuration and observe the NMR signal in the "frozen" local spin configuration (see Fig. 5). The field dependence of the resonance frequency is given by:

$$
\nu=\gamma(H+g\langle s\rangle A)
$$

where the first term is the ${ }^{53} \mathrm{Cr}$ Larmor frequency in the

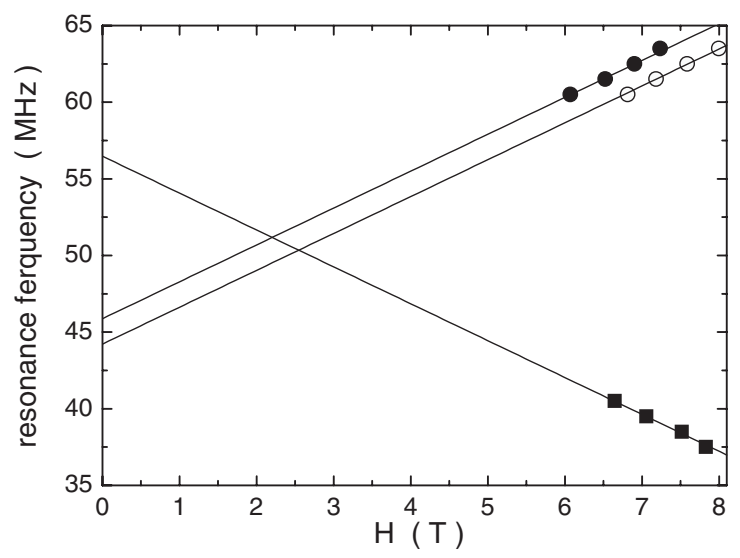

FIG. $2 .{ }^{53} \mathrm{Cr}$ resonance frequency in $\mathrm{Cr} 7 \mathrm{Cd}$ single crystal at $T=1.5 \mathrm{~K}$ as a function of the external applied magnetic field perpendicular to the plane of the molecule.

external field $H$ and the second term is the contribution of the contact field at the nuclear site [12]. Since the average local spin density at low $T$ is constant in the field range investigated 5-10 T (see Fig. 5), one expects a linear field dependence whose slope is given by the gyromagnetic ratio $\gamma$, as observed in Fig. 2. The gyromagnetic ratio extracted from the slope is $2.406 \mathrm{MHz} / \mathrm{T}$, corresponding indeed to ${ }^{53} \mathrm{Cr}$ nucleus.

In order to extract the parameters $A$ and $\langle s\rangle$ from the data it is more convenient to convert the field-swept spectra to a zero field spectrum, as shown in Fig. 3. To operate the conversion, the horizontal axis for the spectrum is changed from the magnetic field to the frequency by subtracting the field contribution at the nuclear site coming from the static external field. In this way the frequencies for the each peak position correspond directly to the internal field at nuclear site, as shown in Eq. (1) with $H=0$.

For the case of a $d$ electron ion like $\mathrm{Cr}^{3+}$ the hyperfine field is dominated by core polarization which yields an internal field opposite to the polarization of the $d$ electron

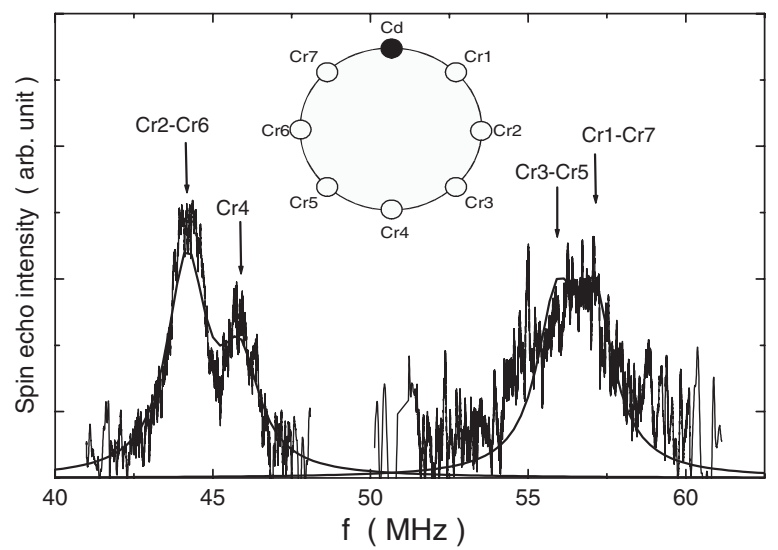

FIG. 3. ${ }^{53} \mathrm{Cr}$ spectrum normalized to zero field (see text for details) in $\mathrm{Cr} 7 \mathrm{Cd}$ at $T=1.5 \mathrm{~K}$. The inset gives the scheme of magnetic ion sites. 
$[13,14]$. The relationship between the local internal field and the spin moment $\langle s\rangle$ is given by Eq. (1) with $A$ given by the negative core polarization field $A_{\mathrm{cp}}$ per $3 d$ electron, i.e., per Bohr magneton and we will assume $g=1.98$.

From symmetry considerations one expects four inequivalent $\mathrm{Cr}$ sites with different spin moments (see inset in Fig. 3). These can be identified as three equivalent pairs (Cr1-Cr7, Cr2-Cr6, Cr3-Cr5) and a single ion (Cr4). From the inspection of the integrated intensity of the NMR lines in Fig. 3, one can assign the single $\mathrm{Cr} 4$ site to the weaker line at $45.87 \mathrm{MHz}(19.06 \mathrm{~T})$. From the plot in Fig. 2 this line has a positive $f$ versus $\mathrm{H}$ slope thus yielding a negative spin polarization $\langle s\rangle$ in Eq. (1) since $A_{\mathrm{cp}}$ is also negative. Because of the antiferromagnetic interaction the polarization of the Cr2-Cr6 pair must also be negative as for $\mathrm{Cr} 4$ and thus they must be identified with the line at $44.22 \mathrm{MHz}$ $(18.38 \mathrm{~T}$ ) in Fig. 3 (see also Fig. 2). The remaining two pairs $(\mathrm{Cr} 1-\mathrm{Cr} 7$ and $\mathrm{Cr} 3-\mathrm{Cr} 5)$ must have positive polarization $\langle s\rangle$ in agreement with the negative slope in Fig. 2. These two pairs yield two NMR lines overlapping into a single broad line (see Fig. 3). The whole spectrum can be fitted with four Lorenzian lines having the same width $(1.5 \mathrm{MHz})$ and intensity proportional to the number of ions per site, i.e., 2:2:2:1. The broad line results from the superposition of two Lorenzian lines centered at $55.9 \pm 0.5 \mathrm{MHz}(-23.3 \pm 0.2 \mathrm{~T})$ and $57.1 \pm 0.5 \mathrm{MHz}$ $(-23.7 \pm 0.2 \mathrm{~T})$. Thus one has four experimental values for the internal field $H_{\text {int }}^{(i)}$ from which one can derive the four different values of the local spin density $\left\langle s_{z}(i)\right\rangle=$ $H_{\mathrm{int}}^{(i)} / g A_{\mathrm{cp}}$, if $A_{\mathrm{cp}}$ is known (since the core polarization depends on core electrons we can assume the same value of $A_{\mathrm{cp}}$ for all $\mathrm{Cr}$ sites). The relevant point here is that $A_{\mathrm{cp}}$ can be obtained from the experiment independently since from magnetic susceptibility one has for the ground state the total spin value $\left\langle S_{z}\right\rangle=3 / 2$. Thus $A_{\mathrm{cp}}=\left[2 H_{\mathrm{int}}^{(1)}+\right.$ $\left.2 H_{\text {int }}^{(2)}+2 H_{\text {int }}^{(3)}+H_{\text {int }}^{(4)}\right] /(1.98 \times 3 / 2)=-12.38 \pm 0.23 \mathrm{~T}$.

The values of the local spin moments assigned to the different $\mathrm{Cr}^{3+}$ spins along the ring for $H$ perpendicular to the plane of the ring are summarized in Table I and are plotted in Fig. 4. It is stressed here that this determination of the staggered nonuniform distribution of local spin moments in the ring is model independent.

We turn now to the theoretical evaluation of the local spin moments to be compared with the experiments in Fig. 4. Low-temperature properties of an AF ring containing $N$ interacting magnetic ions can be described by the following spin Hamiltonian:

$$
\begin{aligned}
\mathcal{H}= & \sum_{i=1, N} J_{i i+1} \mathbf{s}_{i} \cdot \mathbf{s}_{i+1}+\sum_{i=1, N} \sum_{k, q} b_{k}^{q}(i) O_{k}^{q}\left(\mathbf{s}_{\mathbf{i}}\right) \\
& +\sum_{i>j} \mathbf{s}_{i} \cdot \mathbf{D}_{i j} \cdot \mathbf{s}_{j}-\mu_{B} \sum_{i=1, N} g_{i} \mathbf{H} \cdot \mathbf{s}_{i},
\end{aligned}
$$

where $\mathbf{s}_{i}$ are spin operators of the $i$ th magnetic ion in the molecule $\left(\mathbf{s}_{N+1}=\mathbf{s}_{1}\right)$. The first term is the isotropic
TABLE I. Internal field, core polarization hyperfine field, and local spin density determined experimentally for different $\mathrm{Cr}$ sites in $\mathrm{Cr} 7 \mathrm{Cd}$.

\begin{tabular}{lcccc}
\hline \hline Cr site & $H_{\text {int }}($ Tesla) & $A_{\text {cp }}($ Tesla) & $\langle s\rangle$ & $\langle s\rangle$ (theory) \\
\hline Cr1-Cr7 & $-23.7(2)$ & $-12.38(23)$ & $+0.97(8)$ & +1.08 \\
Cr2-Cr6 & $+18.38(4)$ & $-12.38(23)$ & $-0.76(6)$ & -0.84 \\
Cr3-Cr5 & $-23.3(17)$ & $-12.38(23)$ & $+0.95(8)$ & +0.96 \\
Cr4 & $+19.06(8)$ & $-12.38(23)$ & $-0.78(7)$ & -0.87 \\
\hline \hline
\end{tabular}

nearest-neighbor Heisenberg exchange interaction $\left(H_{\text {exc }}\right)$. The second term describes local crystal fields $(\mathrm{CFs})$, with $O_{k}^{q}\left(\mathbf{s}_{\mathbf{i}}\right)$ Stevens operator equivalents for the $i$ th ion [15]. The third term represents the dipolar anisotropic intracluster spin-spin interactions while the last term is the Zeeman coupling with an external field $\mathbf{H}$. In the two systems studied in the present work molecular magnetic anisotropy is almost uniaxial [16,17]. Hence, considering a reference frame with the $z$ axis parallel to the ring axis, the CF term in (2) can be approximately rewritten as $\sum_{i} d_{i}\left[s_{z}^{2}(i)-\right.$ $\left.\frac{1}{3} s_{i}\left(s_{i}+1\right)\right]$, where $s_{z}(i)$ are $z$ components of the spin of the $i$ th magnetic ion. Finally, the axial contribution to the intracluster dipole-dipole interaction $\mathbf{D}_{i j}$ has been evaluated within the point-dipole approximation.

For the AF rings studied here the dimension of the spin Hilbert space is rather large (e.g., 65536 for $\mathrm{Cr} 8$ ). Thus, to diagonalize $\mathcal{H}$, we have followed a two-step procedure [16]. The Heisenberg contribution is largely dominant and the energy spectrum consists of a series of level multiplets with an almost definite value of $|\mathbf{S}|$. Therefore, in the first step of the procedure only the first term in Eq. (2) has been considered. Exploiting the rotational invariance of $H_{\text {exc }}$, the Hamiltonian matrix has been block factorized according to the total spin quantum number $S$ and the corresponding eigenvalues and eigenvectors have been calculated. However, the complete Hamiltonian does not commute with $S^{2}$, hence different $S$ multiplets can mix ( $S$ mixing) and the complete Hamiltonian matrix should be diagonalized [16]. Given that in these systems $S$ mixing is small (at least, far from level anticrossings), we have used the two-

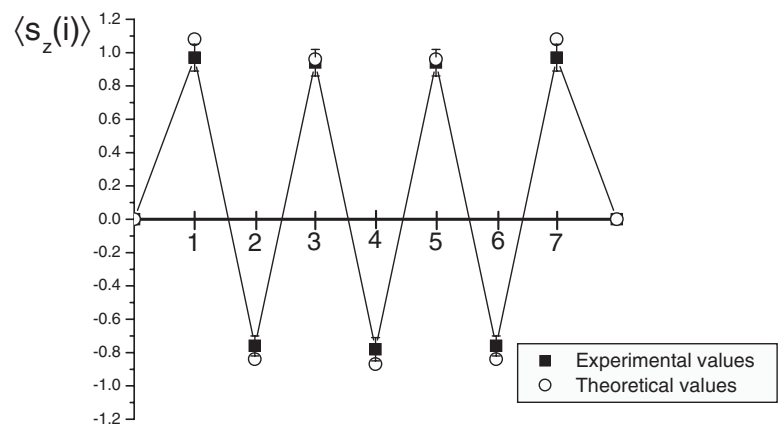

FIG. 4. Experimental (ם) and theoretical ( $\bigcirc)$ values of the local spin densities in $\mathrm{Cr} 7 \mathrm{Cd}$ for $H$ perpendicular to the plane of the ring. 


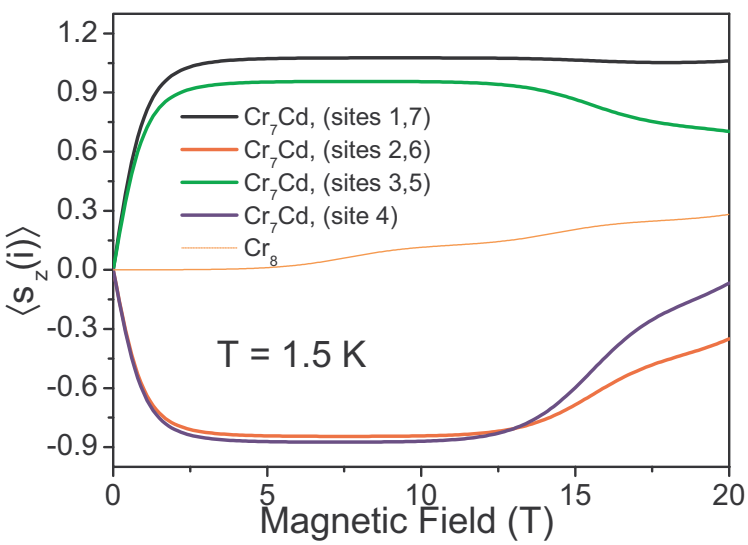

FIG. 5 (color online). Magnetic field dependence of the thermal averages of local spin operators $\left\langle s_{z}(i)\right\rangle$ for $\mathrm{Cr} 8$ and $\mathrm{Cr} 7 \mathrm{Cd}$ $\mathrm{AF}$ rings. The applied field is parallel to the ring axis. $\mathrm{In} \mathrm{Cr} 7 \mathrm{Cd}$, the nonmagnetic $\mathrm{Cd}$ ion is assumed to be on site 8 (see inset Fig. 3). The Hamiltonian parameters have been determined in $[16,17]$.

step perturbative technique described in Refs. $[16,18]$ to include it in the calculation. Calculated eigenstates and eigenvalues have been used to evaluate the magnetic field dependence of the thermal averages of local spin operators $\left\langle s_{\alpha}(i)\right\rangle(\alpha=x, y, z)$. Results are summarized in Fig. 5. For symmetry reasons, far from level crossings in ideal homometallic AF rings $\left\langle s_{\alpha}(i)\right\rangle \simeq S / N$, where $S$ is the total spin of the ground state (Fig. 5). On the contrary, the breaking of the ring symmetry in $\mathrm{Cr} 7 \mathrm{Cd}$ leads to a staggered nature of $\left\langle s_{\alpha}(i)\right\rangle$ and a reduction of the spin value from the $s=3 / 2$ value for a $\mathrm{Cr}^{3+}$ ion. The theoretical results are in good agreement with the experimental values (see Fig. 4). The slight systematic smaller experimental values can be explained by a small delocalization of the $3 d$ orbital of $\mathrm{Cr}^{3+}$ which would reduce the measured experimental spin moment $\langle s\rangle$ and/or by the effective $g$ values.

By concluding, we have shown that by analyzing the NMR spectrum of the nucleus $\left({ }^{53} \mathrm{Cr}\right)$ belonging to the magnetic ion one can determine directly both the core polarization hyperfine constant and the staggered local spin moment $\langle s\rangle$ in the heterometallic $\mathrm{AF}$ ring $\mathrm{Cr} 7 \mathrm{Cd}$. The values obtained for $\langle s\rangle$ are shown to be in good agreement with the theoretical values obtained on the basis of a model Hamiltonian with parameters determined by thermodynamic measurements. This should prove very valuable for the future estimate of local spin moment distribution in heterometallic AF rings of interest for applications such as Cr7Ni having ground state $S=1 / 2$.

Thanks are due to A. Cornia, M.-H. Julien, G. Amoretti, P. Santini, and M. Horvatic for useful comments and suggestions. This work was supported by NoE MAGMANET and RTN QUEMOLNA. Two of the authors (Y.F. and E. M.) thank 21 Century COE programs "Topological Science and Technology" at Hokkaido University for the financial supports.

*Email address: micotti@ fisicavolta.unipv.it

[1] D. Gatteschi et al., Science 265, 1054 (1994).

[2] J. R. Friedman, M.P. Sarachik, J. Tejada, and R. Ziolo, Phys. Rev. Lett. 76, 3830 (1996); see also L. Thomas, F. Lionti, R. Ballou, D. Gatteschi, R. Sessoli, and B. Barbara, Nature (London) 383, 145 (1996).

[3] P. C. E. Stamp, Nature (London) 383, 125 (1996).

[4] D. Gatteschi and R. Sessoli, Angew. Chem., Int. Ed. 42, 268 (2003), and references therein.

[5] M. N. Leuenberger and D. Loss, Nature (London) 410, 789 (2001); see also F. Meier, J. Levy, and D. Loss, Phys. Rev. B 68, 134417 (2003).

[6] F. Troiani, M. Affronte, S. Carretta, P. Santini, and G. Amoretti, Phys. Rev. Lett. 94, 190501 (2005); see also F. Troiani, A. Ghirri, M. Affronte, S. Carretta, P. Santini, G. Amoretti, S. Piligkos, G. Timco, and R. E. P. Winpenny, Phys. Rev. Lett. 94, 207208 (2005).

[7] D. Gatteschi et al., Chemical Society Reviews 25, 101 (1996).

[8] A. Caneschi et al., Chem. Eur. J. 2, 1379 (1996).

[9] F. K. Larsen et al., Angew. Chem. 115, 105 (2003).

[10] J. van Slageren et al., Chem. Eur. J. 8, 277 (2002).

[11] E. Micotti et al. (to be published).

[12] I. Bertini and C. Luchinat, Coord. Chem. Rev. 150, 1 (1996).

[13] A. J. Freeman and R. E. Watson, in Hyperfine Interactions in Magnetic Materials, Magnetism IIA, edited by G. T. Rado and S. Suhl (Academic, New York, 1967).

[14] G. C. Carter, L. H. Bennett, and D.J. Kahan, Metallic Shifts in NMR, Progress in Material Science Vol. 20 (Pergamon, New York, 1977).

[15] A. Abragam and B. Bleaney, Electron Paramagnetic Resonance of Transition Ions (Clarendon, Oxford, 1970).

[16] S. Carretta et al., Phys. Rev. B 67, 094405 (2003).

[17] M. Affronte et al., Appl. Phys. Lett. 84, 3468 (2004).

[18] E. Liviotti, S. Carretta, and G. Amoretti, J. Chem. Phys. 117, 3361 (2002). 